\title{
Roel MEIJER, (ed.), Global Salafism. Islam's New Religious Movement
}

London, Hurst \& Co. Publishers, 2009, 463 p.

\section{Cédric Baylocq Sassoubre}

\section{(2) OpenEdition} Journals

Édition électronique

URL : http://journals.openedition.org/assr/22533

DOI : $10.4000 /$ assr.22533

ISSN : 1777-5825

Éditeur

Éditions de l'EHESS

Édition imprimée

Date de publication : 31 décembre 2010

Pagination : 9-242

ISBN : 9782713223013

ISSN : 0335-5985

Référence électronique

Cédric Baylocq Sassoubre, «Roel MEIJER, (ed.), Global Salafism. Islam's New Religious Movement », Archives de sciences sociales des religions [En ligne], 152 I octobre-décembre 2010, document 152-86, mis en ligne le 10 mai 2011, consulté le 10 décembre 2020. URL : http://journals.openedition.org/ assr/22533; DOI : https://doi.org/10.4000/assr.22533

Ce document a été généré automatiquement le 10 décembre 2020.

(C) Archives de sciences sociales des religions 


\section{Roel MEIJER, (ed.), Global Salafism. Islam's New Religious Movement}

London, Hurst \& Co. Publishers, 2009, 463 p.

\section{Cédric Baylocq Sassoubre}

\section{RÉFÉRENCE}

Roel MEIJER, (ed.), Global Salafism. Islam's New Religious Movement, London, Hurst \& Co. Publishers, 2009, 463 p.

1 En juillet 2009, à l'Assemblée nationale, une Mission d'information sur la pratique du port du voile intégral sur le territoire national était initiée sous la présidence du député André Guérin. Elle aura auditionné deux cents personnes (universitaires, société civile, associatifs...) pour aboutir, le 26 janvier 2010, à un rapport qui préconise une loi d'interdiction de cette pratique dans l'espace public, à laquelle le Conseil d'État n'est pas favorable. Cette commission a mis au premier plan politico-médiatique une branche de l'islam traditionaliste généralement dénommée par le vocable «salafisme». À en juger par les contributions des personnes auditionnées dans le cadre de cette mission, on constate que les contours de cette mouvance protéiforme ne sont pas encore distinctement cernés (toutefois, voir les interventions de M. Doua et S. Amghar: http://www. assemblee-nationale.fr/13/rap-info/i2262.asp). Or, quelques mois après l'ouverture de cette mission paraissait en anglais un ouvrage qui représente la première tentative académique collective pour définir cette mouvance dans la pluralité théologique, juridique et politique de ses expressions transnationales. Global Salafism est une contribution très ambitieuse au champ de l'islamologie en particulier et de l'anthropologie des religions globalisées en général, tiré d'une conférence qui s'est déroulée du 28 au 30 septembre 2007 à l'Université Radboud de Nimègue (Pays-Bas) sous l'égide du regretté International Institute for the Study of Islam in the Modern World de Leyde (qui a fermé ses portes en 2008 après dix ans d'intense activité). Signe du caractère brûlant de la thématique, un essaim de représentants des renseignements 
généraux européens (particulièrement norvégiens, hollandais et allemands) avaient fait le déplacement en ces confins est du pays batave.

Dirigé par Roel Meijer, arabisant et professeur émérite d'histoire du Moyen-Orient à l'Université de Nimègue, l'ouvrage se divise en cinq parties thématiques. La première intitulée Salafist Doctrine s'ouvre avec un article de Bernard Haykel de l'Université de Princeton, qui constitue avec l'introduction de Roel Meijer (pp.1-32), une ressource incontournable pour comprendre la posture fondamentaliste dans l'islam contemporain. Intitulé «On the Nature of Salafi Thought and Action» (pp.33-57), il pose d'abord les topoï communément admis dans le sunnisme classique comme par les chercheurs qui observent ses trajectoires et ses convulsions postmodernes. Il précise: « salafi est prestigieux parmi les musulmans, parce qu'il se réfère à la première et consécutivement authentique version de l'islam - celui des "pieux ancêtres"généralement compris comme étant les trois premières générations de musulmans» et canonisées par un hadith (du recueil de Boukhari) dans lequel le Prophète de l'islam les désignerait comme «les meilleurs de (sa) communauté». En conséquence, ceux qui se réclament de ce minhāj s'inscriraient symboliquement et mimétiquement dans la lignée de ces "pieux prédécesseurs». Haykel relève une autre caractéristique: celle qui s'appuie sur un autre hadith indiquant qu'un seul «groupe» (firqat) de tous ceux qui composent la umma sera sauvé dans l'au-delà, œuvrant ainsi à s'arroger toute la sotériologie islamique. Appropriation du salut que l'on peut retrouver dans certaines branches du judaïsme ou du christianisme, plus précisément chez certaines sectes qui en dérivent. Le discours sotériologique comminatoire n'est donc plus le seul monopole des évangéliques fondamentalistes américains.

3 Mais en bon philologue arabisant cherchant à historiciser l'usage des termes qui labellisent les groupes religieux, il se demande pourquoi «le terme salafi, qui à la fin du dix-neuvième siècle désignait les réformistes modernistes et rationalistes, en est venu à être identifié aux wahhabis pour qui l'argutie religieuse basée sur la raison (aqli) est condamnée.» Comme beaucoup d'autres contributeurs de ce volume, Haykel montre que jusqu'à présent la dimension politique de l'engagement salafiste a été surestimée au détriment des aspects «idéels», «théologiques» et «juridiques» (dans le sens du droit musulman) qui se trouvent au soubassement de leurs conduites.

À cette mise au point d'Haykel, précédée par celle de Meijer, se serait parfaitement articulée cette autre enquête terminologique approfondie de Thomas Hegghammer intitulée "Jihadi-Salafis or Revolutionaries? On Religion and Politics in the Study of Militant Islamism» (pp.244-266). L'équipe éditoriale a choisi de la placer dans la troisième partie, sur laquelle nous reviendrons, consacrée au salafisme-djihadiste ( «Jihadi-Salafism», pp.244-300). L'article de Hegghammer, qui a participé à l'aventure d' Al Qaeda dans le texte (G. Kepel, J.-P. Milelli, dirs., Paris, PUF, 2005), analyse minutieusement les zones de correspondances et les ruptures entre «jihadisme», «takfirisme», et «salafisme». Au terme de son étude, l'emporte la conviction qu'il a réussi son pari d'offrir une grille de lecture plus élaborée de l'«islamisme activiste». Selon lui, se dégagent de cet «islamisme activiste» cinq catégories (que nous avons préféré laisser dans le «jus» anglais de leur élaboration): les mouvements islamistes activistes «State-oriented», les «Nation-oriented», les «Umma-oriented», les «Moralityoriented» et les «Sectarian». Chacune de ces catégories pouvant prendre des formes violentes et non violentes que Hegghammer ne manque pas d'exemplifier. S'il nous est impossible de restituer avec la densité requise cette construction complexe (mais qui, 
au final, simplifie la compréhension de l'«islamisme activiste») nous citerons in extenso le passage où il décrit rapidement ces catégories, indiquant «les raisons majeures pour lesquelles les islamistes agissent»:

5 - l'islamisme «State-oriented » se caractérise par un désir de modifier l'ordre social et politique ;

6 - l'islamisme "Nation-oriented » se définit par un désir d'établir la souveraineté sur un territoire spécifique qui est perçu comme étant occupé ou dominé par des nonmusulmans ;

7 - l'islamisme "Umma-oriented» se distingue par un souci de protéger la Nation musulmane conçue comme un tout, de toute menace (non musulmane) extérieure ;

8 - l'islamisme «Morality-oriented» se caractérise par le désir d'infléchir les comportements musulmans dans une direction plus conservatrice et littéraliste ;

9 - l'islamisme «sectaire» (sectarian Islam) se manifeste par le désir de réduire l'influence et le pouvoir des mouvements concurrents (chiites ou sunnites).

Et de décliner à la suite les formes (violentes ou non) que peuvent prendre les différentes expressions de «l'islamisme activiste».

11 Le chapitre (dont l'article précédent, rappelons-le, ne fait pas partie) se poursuit en toute logique au cœur de la production de l'idéologie salafiste: l'Arabie Saoudite (pp. 58-80). Stéphane Lacroix (chaire Moyen-Orient Méditerranée de Sciences-Po Paris) présente la contribution de Nasir al-Din al-Albani à la pensée salafiste contemporaine. Après un tableau des origines $d u$ wahhabisme, S. Lacroix explique comment cet Albanais, fils de 'alim, né en 1914, est devenu l'un des muhaddif les plus prestigieux d'Arabie Saoudite. Prestigieux, donc controversé, jusqu'à en agacer l'establishment religieux saoudien, quoique "son credo ('aqìda) fût irréprochablement wahhabi» comme ne manque pas de l'indiquer l'auteur, ajoutant qu'en «diverses occasions, les fatwas édictées par al-Albani outragèrent l'institution religieuse». Comble de provocation, il affirma dans son ouvrage sur le hiğăb que les femmes étaient en droit de ne pas se couvrir le visage, ce qui contrevenait directement à la pratique, imposée au royaume saoudien, du niqāb. Où l'on voit que le traditionalisme n'est pas un bloc monomorphe et répond aussi à des enjeux de maintien du pouvoir, assis sur des fondements hanbalite, qu'al-Albani a fait légèrement trembler de l'intérieur. On entrevoit également en quoi il est délicat d'attribuer le qualificatif d'«orthodoxe» aux wahhabis, puisqu'eux aussi s'offrent des libertés d'interprétations avec le Texte sacré comme en témoigne la canonisation de la pratique du niqāb, qui fait tant parler aujourd'hui en Europe. Pour en revenir plus directement à al-Albani, le soutien prudent d'Ibn Baz (lui-même membre de cet establishment) ne lui suffira pas à revenir en grâce. S. Lacroix se concentre ensuite sur un mouvement islamiste saoudien concurrent du wahhabisme, les «neo-Ahl al-Hadith», qui en sont progressivement venus à se réclamer d'al-Albani. Autre glissement infime qu'il est nécessaire de saisir pour ne pas s'embrouiller dans la nébuleuse salafiste. L'intérêt de son article est de montrer comment - fût-ce dans l'antre même du wahhabisme - ce courant n'échappe pas à des repositionnements stratégiques et des dissensions induites non pas seulement par des discussions d'ordres théologico-canoniques mais par des visées politiques (deux ordres entre lesquels ce volume tout entier opère des va-et-vient). Le genre d'étude déconstructiviste qui contribue à nous défaire de l'idée que l'Arabie saoudite est le royaume de l'application des idées pures de l'islam. Au cas où l'on aurait oublié par 
ailleurs que la wahhabiya, d'ovni flottant à la périphérie du sunnisme en est venue quasiment à se confondre avec l'orthodoxie de celui-ci, à la faveur de contingences géopolitique puis géologique.

Et l'article suivant (pp.81-106) enfonce en quelque sorte le clou de cette «relativisation de l'universel» que s'arroge le salafisme puisqu'il présente «La transformation d'un concept radical», qui par l'investissement d'un religieux salafiste (Abu Muhammad alMaqdissi) est passé du statut d'hétérodoxe à celui de pilier de l'engagement du sunnisme orthodoxe (ou de ce qui se présente comme tel). Joas Wagemakers procède à une étude minutieuse du principe d'al walä' wa-l-barā'a («la loyauté et le désaveu»), depuis son émergence tribale antéislamique, jusqu'à la torsion sémantique que lui imprime l'idéologie djihadiste, en passant par son émergence dans le fracas de la géopolitique ancienne de l'islam sous la secte kharijite (à partir des $\mathrm{VII}^{\mathrm{e}}$-VIII ${ }^{\mathrm{e}}$ siècles de notre ère, puis déclinante...) Ce principe prétend condamner tout type non seulement d'«alliance» mais d'amitié ou de proximité entre musulmans et non-musulmans, et entre «bon musulmans» et "mauvais musulmans». Cette enquête philologique et historiographique est non seulement passionnante mais de surcroît salutaire, car, quoiqu'elle fasse montre d'une impeccable neutralité axiologique, elle déconstruit à son tour (et de manière peut-être plus profonde que la contribution précédente) le discours religieux qui se présente comme pur et authentique (ou, quand il est encombré d'un peu de modestie, comme "plus pur» et «plus authentique»). On s'aperçoit que ce discours est en fait, comme d'autres, l'objet de contingences, de distorsions qui répondent à un besoin plus immédiat et prosaïque que spirituel au sens strict (Voir Reza Negarestani, 2006, The Militarization of Peace. Absence of Terror or Terror of Absence? in Mackay R., ed., Collapse: Philosophical Research and Development, vol.1. Oxford, Urbanomic).

13 C'est ensuite vers un stade un peu plus appliqué de l'idéologie salafiste que mène une contribution de Guido Steinberg sur les «racines intellectuelles de l'anti-chiisme» chez les salafistes-djihadistes (pp.107-125). Ce premier chapitre sur la «doctrine salafiste» se clôt par un article de la spécialiste française du Pakistan, Mariam Abou Zahab (pp. 126-142) qui plonge dans la généalogie du mouvement Ahl-e hadith. Il possèderait près de cinq cents madrasas au Pakistan, ce qui en fait un mouvement largement minoritaire dans ce pays par rapport aux déobandis notamment, mais très actif et qui a connu l'expansion la plus importante depuis la fin des années quatre-vingt (+131\%), deux caractéristiques du salafisme à l'échelle transnationale.

Armé de la grille de lecture complète qu'offrent les trois articles de Meijer, Haykel et Heggammer, et des illustrations doctrinales de Lacroix, Wagemakers, Steinberg, Abou Zahab, on peut se lancer plus aisément dans la cartographie planétaire du salafisme. Ainsi le deuxième chapitre «Salafism and Politics» (pp.127-220) s'ouvre sur une étude de Noah Salomon autour du phénomène salafiste au Soudan. Salomon termine une thèse sur les relations entre soufis et salafistes au Soudan, un sujet certainement en devenir, avec celui des relations entre le salafisme et les Frères Musulmans, au vu de la dynamique des relations intra-musulmanes mondiales. Il s'intéresse ici à la "critique salafiste de l'islamisme (comprenez islamisme politique)»(pp.143-168). On est certes impressionné par l'appareil de notes de bas de page qu'il déploie mais elles finissent par prendre le dessus sur le corps de l'article. Néanmoins son article très dense regorge d'entretiens et d'informations de première main provenant de dignitaires salafistes de son terrain, ce qui est assez rare pour être signalé. On y apprend que des mouvements 
salafistes comme Ansar al-Sunna, par exemple, se posent en critiques de l'islamisme politique. L'islamisation de la société passe selon eux plus sûrement par un laborieux travail de terrain prosélyte que par une prise en main par le haut des structures de l'État comme a pu le faire le NIF au Soudan. Ainsi peuvent s'opposer les projets de l'islamisme «moraly-oriented» tel que représenté ici par le mouvement salafiste Ansar al-Sunna, de l'islamisme "state-oriented», à l'instar du NIF, ou encore des Frères musulmans, contre lesquels les salafistes locaux (comme ailleurs) n'ont pas de mots assez durs.

On passe de l'Afrique subsaharienne à l'aire asiatique avec l'enquête de Noorhaidi Hasan sur le mouvement salafiste en Indonésie. Déjà auteur d'une étude très remarquée (Laskar Jihad; Islam, Militancy, and the Quest for Identity in Post-New Order Indonesia, Cornell University, 2006), le chercheur indonésien propose pour commencer son article une solide présentation des penseurs-passeurs transnationaux du salafisme avant de déboucher sur le salafisme indonésien qui s'en inspire ou est directement influencé par eux. À partir de là, Hasan se centre plus sur les divisions intrasalafistes entre Sururis et non-Sururis. La connexion transnationale y apparaît d'emblée évidente, notamment via le salafiste indonésien Ja'far 'Umar Thalib's qui se fait l'élève d'un salafiste yéménite reconnu. L'article montre avec détail comment le salafisme est propice aux multiples fragmentations, la notion de takfïr étant un tuyau d'arrosage à forte pression qui échappe des mains de celui qui le tenait pour arroser tout le monde alentour, y compris lui-même. Le chapitre se clôt avec une riche étude du directeur de l'ouvrage, Roel Meijer, qui se focalise sur l'usage de la notion nécessairement polymorphe d'al-amr bi al-ma'rūf wa an-nahy' 'an al-munkar («prôner ce qui est bien et condamner ce qui est mal») telle que l'a utilisée le mouvement égyptien Gama'a al-islamiyya en guise de levier d'«action sociale» (pp.189-220), et une autre de Khaled Hroub sur la structuration du mouvement salafiste en Palestine, qui, contrairement à ce que l'on pourrait penser, n'a pas pignon sur rue arabe (p.221-243). À tel point qu'il n'a pas vraiment tiré profit de la lutte acharnée entre les frères rivaux du Hamas et du Fatah.

Le chapitre 3 sur le «Salafisme djihadiste», forme la plus radicale du mouvement en question, s'ouvre sur la typologie d'Hegghammer, évoquée plus haut. Dans «Debate within the Family», Reuven Paz passe en revue différentes opinions de «la famille» salafiste-djihadiste sur des thèmes tels que le takfir (au cœur de la prédication salafiste), l'extrémisme, les attentats-suicides et le sens de l'Apocalypse (pp.267-280). Cette étude le mène, en conclusion, à prendre quelque distance avec l'usage du qualificatif salafistedjihadiste auquel il préfère la dénomination de takfiri-djihadiste (jihadi-takfiri). Puis une figure relativement récente de doctrinaire du radicalisme nous est présentée par Bynjar Lia en la personne d'Abu Mus'ab al-Suri (pp.281-300).

17 Le chapitre 4 rentre dans le vif du caractère transnational du salafisme (déjà bien traité avec Noorhadi Hasan), avec Madawi al-Rasheed, Laurent Bonnefoy, et Terje Ostebo qui traitent respectivement de la maison mère saoudienne («The Local and the Global in Saudi Salafi-Jihadi Discourse», pp.301-320), de sa progéniture limitrophe («How Transnational is Salafism in Yémen?» pp.321-341), et de son développement dans un pays d'Afrique subsaharienne assez isolé du reste du monde islamique jusqu'au début des années quatre-vingt («Growth and Fragmentation. The Salafi Movement in Bale, Ethiopia», pp.342-363).

18 Le cinquième et dernier chapitre sur lequel nous nous arrêterons s'intitule «Salafism and Identity». Il aurait pu s'intituler «Salafism in Europe» puisque ce sont trois pays 
européens qui occupent les trois derniers contributeurs: la France, le Royaume-Uni et les Pays-Bas, où ce courant a gagné du terrain ces dernières années. On commence par la France avec Mohammed Ali Adraoui, qui distingue une présence salafiste plus visible qu'ailleurs dans les banlieues de Paris, Lille-Roubaix, et Lyon. Dans sa définition de l'objet salafisme en France, Adraoui articule la position sociale des salafistes à la construction de leur identité religieuse et la conception de leur rapport à la Nation qui en résulte: «Si par exemple dans leur vie passée, Paris représentait le pouvoir culturel, politique et économique de la France, dans leur nouvelle vie elle est devenue ville de kufr (impiété); les personnes et les institutions puissantes qui les rejetaient préalablement sont maintenant vues comme amorales et illégitimes, depuis leur nouveau piédestal exalté. Leur propre communauté a remplacé la désormais allogène (alien) nation française et l'islam a supplanté un Occident antagonique. Les voisins deviennent des mécréants ( $k u f f a ̈ r)$ et les musulmans non salafistes sont déviants.». Adraoui passe en revue d'autres caractéristiques de ce salafisme intra-européen en situation de minorité de la minorité, comme leur attraction vers les pays du Golfe (pp. 368-371). Ici une ethnographie de cours à distance, de récits de voyages ou de références religieuses aurait été bienvenue pour illustrer cette description générale de la propension exotique du mouvement salafiste français (alors que le mouvement frériste est plus enclin à l'inscription dans un contexte national voir local...) Si l'on suit l'auteur dans l'idée que le salafisme («non révolutionnaire») est ici un postislamisme en ce qu'il récuse la politique ou même la recherche de l'acquisition d'un pouvoir politique et a «développé un nouveau type d'idéologie basée sur le besoin de devenir quelqu'un, à savoir l'émancipation sociale en montant une affaire et en acquérant prestige social et respectabilité au sein de leur propre communauté», on peut remarquer que ce ne sont pas là des caractéristiques spécifiquement salafistes. La majorité des jeunes musulmans de France, qu'ils ne se reconnaissent dans aucun mouvement, ou qu'ils adhèrent à une structure associative d'inspiration Frères musulmans ne cherchent pas, eux non plus, à subvertir islamiquement les structures de l'État. Une fois passé le cap d'un jeu d'une réaction/contre-réaction avec la société majoritaire, nombreux sont ceux qui cherchent dans le recours à leur spiritualité un développement personnel et un rapport à l'autre plus apaisé. Le salafisme en revanche montre une certaine âpreté à maintenir un «eux» et un «nous» exclusifs. C'est peut-être en ce sens que ceux qu'Adraoui nomme les salafistes puristes (certainement équivalents de salafis-piétistes, par opposition aux salafis-djihadistes) connaissent malgré tout une forme de politisation de leurs conduites et discours.

Puis Sadek Hamid présente le panorama salafiste en Grande-Bretagne, auquel la sociologie et l'histoire de l'immigration, provenant principalement du sous-continent indien, donnent des caractéristiques propres. En effet, si l'on met en regard le nombre peu élevé de ses citoyens de confession musulmane (moins de trois millions) et son statut de plaque tournante du radicalisme en Europe (Le Français Abd Samad Moussaoui avait raconté dans Zacarias Moussaoui, mon frère, Denoël, Paris, 2002, comment le parcours de son frère, natif de Saint-Jean-de-Luz, s'était infléchi vers le Londonistan, d'où il s'était ensuite engagé dans la préparation des attentats du 11 septembre 2001), le pays se prête particulièrement à une analyse du salafisme (dont nous avons toutefois mentionné plus haut qu'il pouvait exister en une variante quiétiste et même critique de la violence politique...) Dans «The Attraction of Authentic Islam. Salafism and British Muslim Youth» (pp.384-403), Hamid se livre à une sociohistoire du salafisme en Grande-Bretagne d'autant plus intéressante que la genèse 
de ce mouvement est bien plus lisible en Angleterre qu'en France. Effet conjugué de deux caractéristiques très anglo-saxonnes: un mode d'organisation et de communication très bien structuré du salafisme britannique, et un libéralisme religieux étatique qui ne bride pas la visibilité religieuse et laisse libre cours aux discours les plus radicaux (état de fait qui tend toutefois à se modifier depuis les attentas de juillet 2005 à Londres...) En témoignent la mosquée de Green Lane, la mosquée Ibn Taymiyyah de Brixton, l'institut salafiste de Birmingham, et le centre islamique de Luton, quoiqu'Hamid précise bien que tous les centres de prédications salafistes sont loin de se présenter ouvertement comme tels. Hamid liste ensuite deux mouvements qui ont activement participé à répandre l'idéologie salafiste outre-Manche: la Jamiyyah Ihya' Minhaj as Sunnah (JIMAS - Groupe pour le renouveau de la voie prophétique) et le Harakat Islah al-Shabab al-Muslimin (Mouvement de réforme de la jeunesse musulmane). Évidemment, ils se posent en s'opposant vivement aux deux expressions majoritaires de l'islam britannique: les déobandi et les barelwi. Hamid n'oublie pas de rappeler, ce qui est trop souvent ignoré dans quasiment tous les exercices politiques ou journalistiques de condamnation du radicalisme (à connotation) islamique, la distanciation de certaines branches du salafisme avec le terrorisme - particulièrement al-Qaeda qu'ils désignent comme des «kharijites» ou «néo-kharijites» - depuis le 11 septembre, mais plus particulièrement en Grande-Bretagne depuis les attentas de Londres. À ce titre le revirement «loyaliste» d'Abu Muntasir, qui préside aux destinées de la radicale JIMAS est très intéressant (pp.399-400). Reste à savoir si ce revirement consiste à donner seulement l'aspect d'une «friendly-faced voluntary organisation» où si le changement est plus profond, idéologique, théologico-canonique. Ajoutons à l'éclairage de Sadek Hamid que, puisque l'islam britannique, dans un contexte multiculturel revendiqué et des liens transnationaux inévitables, a parti lié avec l'Inde et le Pakistan (plus que la France avec l'Algérie et le Maroc), on ne peut éviter de porter le regard vers ce qui, de ce point de vue, émerge d'Asie Centrale. Récemment, par exemple, le pakistanais Muhammad Tahir ul-Qadri a publié une fatwa argumentée en plus de six cents pages qui condamne la violence politico-religieuse. Aussitôt, son travail a été traduit en anglais (Shaykh ul-Islam Dr Muhammad Tahir ul-Qadri, Fatwa on Suicide Bombings and Terrorism, Minhaj Publications, Minhaj-ul-Quran International, Londres, février 2010, accessible sur www. minhaj.org) et circulait, que ce soit dans les milieux soufis (proche du cheikh en question), dans les milieux fréristes (Muslim Association of Britain) et salafistes «repentis» (JIMAS) ou dans le milieu associatif transeuropéen des jeunes cadres musulmans pacifistes (Forum for Muslim Youth in Europe, FEMYSO)... Malheureusement cette fatwa positive n'a pas fait force de loi chez les talibans qui ont assassiné, en mai 2010, soixante-dix membres de la communauté ahmadiyya qui priaient paisiblement dans leur mosquée le vendredi.

Enfin, l'ethnographe sera soulagé que le recueil d'articles se termine par l'étude de Martjin de Koning («Changing Worldviews and Friendship. An Exploration of the Life Stories of Two Female Salafis in the Netherlands», pp.404-423) tant la succession de textes d'idéologues salafis, de discours ex-cathedra, et d'analyses géopolitiques avaient quelque peu relégué l'échelle locale et interindividuelle. Or, c'est précisément à cette échelle que les jeunes Européens de confession musulmane qui se réclament du salafisme s'approprient ces textes et discours transnationaux.

21 Cette approche du salafisme européen du point de vue féminin est cruciale tant, comme l'explique parfaitement de Koning «Les jeunes femmes ont une position importante dans les politiques de l'identité des jeunes musulmans parce qu'elles sont responsables 
de la reproduction de la culture de leurs parents et de l'islam. Plus que les jeunes hommes, elles sont en permanence scrutées par les autres Hollando-Marocains ainsi que par les Hollandais d'ascendance hollandaise "native-Dutch"), en particulier leur comportement et leur manière de se vêtir.» Que ce soit dans les sociétés sécularisées où les sociétés cléricalisées, la femme est un «enjeu» presque aussi majeur que le territoire. Un enjeu quasi géopolitique. Topographie naturelle, urbaine... et féminine. On suit donc pas à pas les trajectoires de ces deux jeunes femmes. Le récit est trop riche pour être résumé ici, mais on notera que, bien que déjà musulmane avant de passer au salafisme, ces jeunes femmes considèrent leur basculement dans ce type de piétisme comme une véritable "conversion». Ce que confirme une enquête récente coréalisée (Cédric Baylocq Sassoubre, Akila DriciI-Bechikh, «The Salafis and the Others. An Ethnography of Intra-Communal Relations in French Islam», in Dupret B., Pierret Th., Pinto P., Spellmann K., (éds), Ethnographies of Islam, Londres-Edimburgh, Agha Khan/ Edimburgh University Press, à paraître).

On pourrait dire, pour être un peu plus complet, qu'un dix-neuvième contributeur plane au-dessus de cet ouvrage tant il est cité. Il s'agit de Quintan Wikctorowicz, convoqué par de nombreux contributeurs pour son maitre article "Anatomy of the Salafi Movement» (Studies in Conflict and Terrorism, 29-3, 2006, pp.207-239) pour "The New Global Threat: Transnational Salafis and Jihad» (Middle East Policy, 8-4, 2001, pp. 18-38). Deux auteurs en signalent les limites (Haykel, Heggammer), les autres mentionnent la typologie comme effort de compréhension intéressant ou s'appuient carrément dessus.

Il ne faudrait pas terminer sans mentionner que l'ouvrage est agrémenté d'un très utile glossaire des termes récurrents du salafisme au début et d'une série de fiches biographiques de penseurs et leader salafistes à la fin. L'accumulation de ces articles de haute tenue, d'experts majoritairement arabisants, ainsi que les tentatives réussies de distinguer et classifier, les différentes expressions de la nébuleuse salafiste font que cet ouvrage s'impose au final comme un vade-mecum de la géopolitique de l'islam et un ouvrage important des sciences religieuses contemporaines.

$\mathrm{Au}$ regard des capacités analytiques de l'équipe, on se prend toutefois à regretter qu'une étude comparative systématique avec les langages totalitaires n'ait pas été entreprise, afin de caractériser un peu plus les spécificités ou au contraire les permanences du salafisme à l'échelle transhistorique des mouvements radicaux. Le salafisme (ou plutôt telle expression salafiste qu'il conviendrait de circonscrire avant de se prononcer) est-il un néofascisme ou pas? À ce titre on trouvera dans l'œuvre de Jean-Pierre Faye (Langages totalitaires, Critique de la raison et de l'économie narrative, Paris, Hermann, 2004; Introduction aux langages totalitaires. Théorie et transformations du récit, Le Livre de Poche, 2009) ainsi que dans celle de Viktor Klemperer (LTI, la langue du Troisième Reich. Carnets d'un philologue, Paris, Albin Michel, 1996), ou de George Mosse (La Révolution fasciste. Vers une théorie générale du fascisme, Paris, Seuil, 2003) des éléments possibles de comparaison avec la langue et les récurrences du III Reich. Il ne sera pas possible de déterminer si telle composante du salafisme est de type fasciste avant d'avoir réalisé ce travail systématique. Néanmoins c'est l'autre mérite du présent ouvrage que d'inaugurer avec sérieux tout un nouveau pan du champ de l'islamologie. 